\title{
miR-219-3p regulates the occurrence of hepatic fibrosis by targeting Smad2
}

\author{
WEIHUA YIN, WEI ZHANG, YANFANG ZHU, HUIHUI NI, LI GONG and MAOYING FU \\ Department of Infectious Diseases, The First People's Hospital of Kunshan Affiliated with Jiangsu University, \\ Suzhou, Jiangsu 215000, P.R. China
}

Received June 13, 2018; Accepted February 5, 2019

DOI: $10.3892 /$ etm.2019.7480

\begin{abstract}
Abnormal expression of microRNA (miR)-219-3p has been widely identified in different tumors. However, whether miR-219-3p is involved in the progression of hepatic fibrosis (HF) has never been explored. The present study showed that compared with healthy controls, the levels of miR-291-3p in peripheral blood were decreased in patients with HF. Furthermore, much lower levels of miR-291-3p were identified in fibrotic liver tissues compared with that of normal liver tissues. Receiver operating characteristic curve analysis showed that the levels of miR-291-3p in peripheral blood may screen patients with HF from healthy controls. Reverse transcription quantitative polymerase chain reaction analysis showed that overexpression of miR-291-3p significantly suppressed the mRNA levels of Snai1, vascular endothelial-specific cadherin (VE-cadherin), Vimentin, transforming growth factor(TGF)- $\beta 1$, and glial fibrillary acidic protein (GFAP). The protein levels of Snai1, VE-cadherin, Vimentin, TGF- $\beta 1$, and GFAP were also decreased in hepatic stellate cells transfected with miR-291-3p mimics. Further study indicated that mothers against decapentaplegic homolog 2 (Smad2) was a target gene of miR-291-3p. More importantly, silencing of Smad2 could abolish miR-291-3p inhibition-induced TGF- $\beta 1$ signaling activation. In summary, reduced peripheral blood miR-291-3p may be involved in the progression of HF via targeting Smad2.
\end{abstract}

\section{Introduction}

Hepatic fibrosis (HF) is a chronic liver disease, and a major cause of morbidity and mortality worldwide (1). HF can be induced by viral hepatitis, alcohol/nonalcoholic steatohepatitis

Correspondence to: Dr Maoying Fu, Department of Infectious Diseases, The First People's Hospital of Kunshan Affiliated with Jiangsu University, 91 Qianjing West Road, Suzhou, Jiangsu 215000, P.R. China

E-mail:ww20180528@yeah.net

Key words: microRNA-291-3p, hepatic fibrosis, mothers against decapentaplegic homolog, transforming growth factor $\beta 1$ signaling and several other etiologies, which then cause persistent damage and stimulation (2). In the development of HF, an enhanced fibrotic matrix is identified, which is due to the disturbance of extracellular matrix (ECM) synthesis and degradation (3). With the progression of HF, it may further result in liver cirrhosis and hepatocellular carcinoma, inducing a major public health concern (4). Hence, it is particularly important to explore an effective therapy method for patients with HF.

Hepatic fibrosis is a pathological process in which a large number of activated fibroblasts are produced in the liver, resulting in excessive precipitation of diffuse extracellular matrix in the liver (5). In recent years, it has been identified that hepatocytes and bile duct epithelial cells can be transformed into fibroblasts through the Epithelial-Mesenchymal Transition (EMT) process and participate in the development of liver fibrosis (6). Lim et al (7) reported that the hepatic stellate cell (HSC) may be an epithelial cell in resting state. After HSC was activated, E-cadherin was transformed into $\mathrm{N}$-cadherin, a marker of interstitial cells (8). These results suggest that HSC can form fibroblasts through EMT activation and participate in the occurrence of liver fibrosis (8). Hence, the present study aimed to examine the changes of EMT markers that are closely associated with liver fibrosis.

Transforming growth factor (TGF)- $\beta$ is a key regulator in chronic liver disease where it promotes the process of fibrogenesis through inflammation (9). Within the liver, TGF- $\beta$ is secreted by platelets and Kupffer cells upon stimulation of the inflammatory response (10). A significant enhancement in TGF- $\beta$ expression is identified in the activated hepatic stellate cells, which is accompanied by the accelerated accumulation of ECM (11). In general, TGF- $\beta$ exerts its biological function via activating downstream regulators, mothers against decapentaplegic homolog (SMAD) 2 and 3 (12). Subsequently, SMADs interact with other signaling pathways, including the mitogen-activated protein kinase (MAPK) and nuclear factor $(\mathrm{NF})-\kappa \mathrm{B}$ signaling pathways, thereby driving the process of hepatic fibrosis (12).

Currently, increasing evidence has indicated that microRNAs (miRs) are widely involved in TGF- $\beta$-mediated liver fibrosis $(13,14)$. For instance, miR-130a-3p is demonstrated to be negatively regulating HSC activation and proliferation in the development of nonalcoholic steatohepatitis by suppressing the expression TGFBR1 and TGFBR2 via the TGF- $\beta /$ SMAD 
signaling pathway (15). Additionally, miR-9-5p was shown to inhibit TGF- $\beta 1$-induced HSC activation via targeting TGFBR1 and TGFBR2 (16). Abnormal expression of miR-291-3p has been widely identified in different tumors, including malignant melanoma and colorectal carcinoma $(17,18)$. A previous study has indicated that miR-291-3p induces liver cell apoptosis via targeting RNA binding protein HuR (19). Additionally, increased miR-291-3p expression levels led to abnormal glucose and lipid metabolism in liver cells $(20,21)$. These data suggested that abnormal miR-291-3p level in liver cells could result in liver cell damage. However, whether miR-291-3p is involved in the progression of HF has never been explored. In the present study, the expression and functional role of miR-291-3p in the development of liver fibrosis was evaluated for the first time, to the best of our knowledge.

\section{Patients and methods}

Human specimens. Peripheral blood or liver tissue were collected from a total of 40 patients diagnosed with posthepatitic cirrhosis and admitted into First People's Hospital of Kunshan Affiliated with Jiangsu University from February to October in 2017. These included 22 males and 18 females with an average age of $52.5 \pm 13.4$ years. Two of them were diagnosed at stage I, three were at stage II, 10 were at stage III, and 25 were at stage IV. Another 40 healthy volunteers from the physical examination center of the hospital were enrolled as the normal control group, including 24 males and 16 females with an average age of $43.6 \pm 14.5$ years. The normal liver samples were obtained from residual liver tissues because of technical reasons (the fibrotic tissues were harvested from an improper location). Normal liver tissues were determined using immunohistochemical analysis. Liver cirrhosis was diagnosed by liver biopsy and/or a typical appearance of the liver on abdominal ultrasound and/or computed tomography scan. Informed consent for use of liver samples was obtained from all participants. Peripheral blood $(10 \mathrm{ml})$ was collected from 40 healthy control and 40 patients with liver cirrhosis. The present study was approved by the Ethics Committee of the First People's Hospital of Kunshan Affiliated with Jiangsu University (KSAJSU-20160819) and was performed in compliance with the Declaration of Helsinki. All the patients have provided written informed consent for this study. The details for the control and patients with liver cirrhosis were included in Table I.

Isolation and culture of rat hepatic stellate cells (HSCs). Adult male Sprague-Dawley rats (body weight, 400-500 g; age, 6-8 weeks) were purchased from Shanghai Silaike Experimental Animal Limited Liability Company (Shanghai, China) and were used for HSC isolation, as previously described (22). Rats were maintained in the animal experimental center of Wenzhou Medical University and four animals were housed per cage with a $12 \mathrm{~h}$ light/dark cycle. Room temperature was maintained at $23 \pm 1^{\circ} \mathrm{C}$, humidity was maintained at $\sim 60 \%$ and all rats had free access to food and water. The present study was approved by the Animal Ethics Committee of the First People's Hospital of Kunshan Affiliated with Jiangsu University (KSAJSU-20160819). Cells were cultured in
Dulbecco's modified Eagle's medium (DMEM; Hyclone; GE Healthcare Life Sciences, Logan, UT, USA) supplemented with $10 \%$ fetal bovine serum (Invitrogen; Thermo Fisher Scientific, Inc., Waltham, MA, USA), 100 U/ml penicillin and $100 \mathrm{U} / \mathrm{ml}$ streptomycin. The harvested primary HSCs were studied at day 0 after isolation throughout all the studies. Primary HSCs at a density of $10^{6}$ cells/well were transfected with miR-291-3p mimics/inhibitors or the negative control for miR-291-3p mimic (NC)/miR-291-3p inhibitors (NCi; both Shanghai GeneChem, Co., Ltd., Shanghai, China) at $37^{\circ} \mathrm{C}$ for $48 \mathrm{~h}$ using Lipofectamine RNAiMAX (Invitrogen, Thermo Fisher Scientific, Inc. Waltham, MA, USA) at a final concentration of $10 \mathrm{nM}$. Following $48 \mathrm{~h}$ of transfection, cells were collected for subsequent experimentation.

Transfection of siRNA targeting Smad2. Primary HSCs at the density $10^{6}$ cells/well were transfected with siRNA targeting Smad 2 or an NC (both Shanghai GeneChem Co., Ltd.) at $37^{\circ} \mathrm{C}$ for $48 \mathrm{~h}$ using Lipofectamine RNAiMAX (Invitrogen; Thermo Fisher Scientific, Inc.) at a final concentration of $10 \mathrm{nM}$. Following $48 \mathrm{~h}$ of transfection, cells were collected for subsequent experimentation.

Cell culture. 293T cells were purchased from the Chinese Academy of Medical Sciences (Beijing, China). Cells were cultured in DMEM/F12 (GE Healthcare Life Sciences) supplemented with $10 \%$ fetal bovine serum, $100 \mathrm{U} / \mathrm{ml}$ penicillin and $100 \mathrm{U} / \mathrm{ml}$ streptomycin in $25 \mathrm{~cm}^{3}$ culture flasks at $37^{\circ} \mathrm{C}$ in a humidified atmosphere containing $5 \% \mathrm{CO}_{2}$.

Transwell migration assay. Cells were placed in the top chamber of Transwell migration chambers $(8 \mu \mathrm{m}$; Millipore, Billerica, MA, USA) at a density of $10^{5}$ cells/well in $1 \mathrm{ml}$ DMEM culture. After $48 \mathrm{~h}$, cells which had not migrated to the lower chamber were removed from the upper surface of the Transwell membrane with a cotton swab. Migrating cells on the surface of the lower membrane were fixed with methanol for $15 \mathrm{~min}$ at room temperature. Methanol was then discarded and washed using PBS. The cells were further stained with $0.5 \%$ crystal violet for $20 \mathrm{~min}$ at room temperature. Cells were subsequently imaged and counted using a light microscope (XDS-500D; Shanghai Caikon Optical Instrument Co., Ltd., Shanghai, China) with 100x magnification. Experiments were assayed in triplicates, and $\geq 5$ fields were counted in each experiment.

Proliferation assay. Cell proliferation was evaluated using the cell counting kit-8 (CCK-8; Dojindo, Kumamoto, Japan), according to the manufacturer's protocol. Cells were seeded in 96-well plates at a density of $1 \times 10^{3}$ cells per well and cultured for $24 \mathrm{~h}$ at $37^{\circ} \mathrm{C}$. Subsequently, cells were transfected with miR-291-3p mimics. After 48 h, CCK-8 solution was added to each well in the 96 -well plates. Then, cells were incubated for an additional $2 \mathrm{~h}$ at $37^{\circ} \mathrm{C}$. Absorbance was determined at $450 \mathrm{~nm}$ on a microplate reader (Molecular Devices, Sunnyvale, CA, USA).

Reverse transcription-quantitative polymerase chain reaction $(R T-q P C R)$. RNA was isolated from liver tissues, 
Table I. Clinical characteristics of healthy control and patients with liver cirrhosis.

\begin{tabular}{lcr}
\hline Characteristic & Control $(\mathrm{n}=40)$ & Liver cirrhosis $(\mathrm{n}=40)$ \\
\hline Sex (male/female) & $24 / 16$ & $22 / 18$ \\
Age (years) & $43.6 \pm 14.5$ & $52.5 \pm 13.4$ \\
Tumor stage (n) & & 2 \\
Stage I & & 3 \\
Stage II & & 10 \\
Stage III & & 25 \\
Stage IV & & $106.7 \pm 157.9^{\mathrm{a}}$ \\
TBIL ( $\mu$ B) & $11.8 \pm 3.8$ & $122.4 \pm 235.1^{\mathrm{a}}$ \\
ALT (U/l) & $21.3 \pm 5.6$ & $116.4 \pm 182.5^{\mathrm{a}}$ \\
AST (U/l) & $20.3 \pm 4.8$ & $32.5 \pm 8.1^{\mathrm{a}}$ \\
ALB (g/l) & $46.8 \pm 2.7$ & $116.4 \pm 138.9^{\mathrm{a}}$ \\
GGT (U/l) & $23.5 \pm 8.2$ & $163.2 \pm 96.5^{\mathrm{a}}$ \\
AKP (U/l) & $56.2 \pm 8.4$ & $18.3 \pm 13.6^{\mathrm{a}}$ \\
PT time (sec) & $12.1 \pm 0.5$ & $6.7 \pm 15.9^{\mathrm{a}}$ \\
HBV DNA level & & \\
\hline
\end{tabular}

${ }^{\mathrm{a}} \mathrm{P}<0.05$ vs. the control. AKP, alkaline phosphatase; ALB, albumin; ALT, alanine transaminase; AST, aspartate aminotransferase; GGT, glutamyltransferase; HBV, hepatitis B virus; PT, prothrombin time; TBIL, total bilirubin.

peripheral blood or primary HSCs using RNA TRIzol reagent (Life Sciences; Thermo Fisher Scientific, Inc.) following the manufacturer's protocol. Total RNA was subsequently reverse transcribed to cDNA using SuperScript ${ }^{\mathrm{TM}}$ III Reverse Transcriptase (Invitrogen; Thermo Fisher Scientific, Inc.) following the manufacturer's protocol. qPCR was performed using SYBR-Green PCR Master Mix (Roche Diagnostics, Basel, Switzerland) on an Applied Biosystems ViiA 7 real-time PCR system (Thermo Fisher Scientific, Inc.). The final reaction volume was $10 \mu \mathrm{l}$ and contained $5 \mu \mathrm{l}$ SYBR-Green PCR Master Mix (2X), $0.5 \mu \mathrm{l}$ forward and $0.5 \mu \mathrm{l}$ reverse primers (10 mM), $2 \mathrm{ml} \mathrm{cDNA}$ and $2 \mu \mathrm{l}$ double-distilled water. The thermocycling conditions of qPCR were: Denaturation at $95^{\circ} \mathrm{C}$ for $10 \mathrm{~min}$, followed by 40 cycles of $95^{\circ} \mathrm{C}$ for $10 \mathrm{sec}$ and $60^{\circ} \mathrm{C}$ for $60 \mathrm{sec}$. The results were normalized to U6 expression to obtain $\Delta \mathrm{Cq}$ values and fold changes in expression were calculated using the $2^{-\Delta \Delta \mathrm{Cq}}$ method (23).

The primers used in the current study were listed as follows: Snail forward, 5'-GCAGAGTTGTCTACCGAC CT-3' and reverse, 5'-AGGTGAACTCCACACACGC-3'; VE-cadherin forward, 5'-CCAGAATTTGCCCAGCCC TA-3' and reverse 5'-GTCCTCGTTCTTCAGGGCAA-3'; Vimentin forward, 5'-TCCTTCGAAGCCATGTCCAC-3' and reverse, 5'-GTGGTCACATAGCTCCGGTT-3'; TGF- $\beta 1$ forward, 5'-GACTCTCCACCTGCAAGACC-3' and reverse, 5'-GGACTGGCGAGCCTTAGTTT-3'; glial fibrillary acidic protein (GFAP) forward, 5'-TTGACCTGCGACCTTGAG TC-3' and reverse, 5'-GAGTGCCTCCTGGTAACTCG-3'.

Dual luciferase reporter assay. MiRNAs targets were predicted using the TargetScan website (www.targetscan.org). Wild-type (WT) and mutant seed regions of miR-291-3p in the 3'-untranslated region (UTR) of the Smad2 gene were chemically synthesized in vitro, XhoI and NdeI restriction sites were added, and then cloned into pmirGLO luciferase reporter vector (Promega Corporation, Madison, WI, USA).

Prior to conducting the dual reporter assay, $5 \times 10^{4} 293 \mathrm{~T}$ cells/well were seeded in 24-well plates with $500 \mu$ l DMEM and cultured for $18 \mathrm{~h}$ at $37^{\circ} \mathrm{C}$. The cells were transfected with the modified firefly luciferase reporter vector $(500 \mathrm{ng} / \mu \mathrm{l})$ mixed with Vigofect transfection reagent (Vigorous, Beijing, China), according to the manufacturer's protocol. After continuous exposure of miR-291-3p/pmirGLO-Smad2-3'UTR or $\mathrm{NC} /$ pmirGLO blank vector for $48 \mathrm{~h}$, the luciferase activities of firefly and Renilla were measured with the Dual-Luciferase ${ }^{\circledR}$ Reporter Assay System (Promega Corporation), according to the manufacturer's protocol. Firefly luciferase activity was normalized to Renilla luciferase activity.

Western blotting. Liver tissues or primary HSCs were treated with radioimmunoprecipitation assay buffer containing $1 \%$ (v/v) phenylmethylsulfonyl fluoride (both Beijing Solarbio Science and Technology Co., Ltd., Beijing, China), 0.3\% (v/v) protease inhibitor (Sigma-Aldrich; Merck KGaA, Darmstadt, Germany) and $0.1 \%$ (v/v) phosphorylated proteinase inhibitor (Sigma-Aldrich; Merck KGaA). A bicinchoninic protein assay kit (Pierce; Thermo Fisher Scientific, Inc.) was used to determine the protein concentration. Subsequently, supernatants were extracted from the lysates following centrifugation at $11,000 \mathrm{xg}$ at $4^{\circ} \mathrm{C}$ for $15 \mathrm{~min}$. Equal amounts of protein ( $30 \mu \mathrm{g} /$ lane) were separated using 10\% SDS-PAGE at $300 \mathrm{~mA}$ for $2 \mathrm{~h}$ and transferred onto a polyvinylidene fluoride membrane, as previously reported (10). The following primary antibodies were used: $\beta$-Actin (cat. no. 4970), Snail (cat. no. 3879), vascular endothelial cadherin (VE-cadherin; cat. no. 2500), Vimentin (cat. no. 5741), TGF- $\beta 1$ (cat. no. 3711), GFAP (cat. no. 80788; all 1:1,000; Cell 
Signaling Technology, Inc., Danvers, MA, USA). HSCs are the main contributors of hepatic fibrosis (24). Glial Fibrillary Acidic Protein (GFAP), first identified in astroglial cells, belongs to intermediate filaments, which preserves cell's mechanical strength and structure (25). TGF- $\beta$-mediated activation of GFAP has been extensively reported $(26,27)$. A previous study has demonstrated that GFAP could be a more useful marker of early Hepatic Stellate Cells (HSC) activation than $\alpha$-SMA (24). It is suggested that GFAP may act as a mesenchymal marker of early HSCs activation in liver fibrosis (28). Hence, the present study evaluated the expression of GFAP. Following several washes with TBS with Tween (TBST), the membranes were incubated with horseradish peroxidase (HRP)-conjugated goat anti-rabbit IgG (1:5,000; cat. no. ZB-2306, Zhongshan Gold Bridge Biological Technology Co., Beijing, China) for $2 \mathrm{~h}$ at room temperature and then washed. The proteins were detected using enhanced chemiluminescence, according to the manufacturer's protocol (Merck KGaA). ImageJ 1.8.0 (National Institutes of Health, Bethesda, MD, USA) was used to quantify the relative protein levels. $\beta$-Actin was used as an internal control.

Statistical analysis. The data are represented as the mean \pm standard deviation (SD). Two-tailed unpaired Student's t-tests were used for comparisons of two groups. Comparisons of means among multiple groups were determined using one-way analysis of variance followed by a Turkey's post-hoc test. Receiver operating characteristic (ROC) curves were used to assess miR-291-3p as a biomarker and the area under the curve was reported (version 20.0; SPSS, Inc., Chicago, Illinois). $\mathrm{P}<0.05$ was considered to indicate a statistically significant difference.

\section{Results}

Decreased miR-291-3p in the peripheral blood and liver tissues of patients with HF. First, the levels of miR-291-3p in the peripheral blood and liver tissues of patients with HF were detected. Compared with healthy controls $(1 \pm 0.69)$, the levels of miR-291-3p in the peripheral blood were decreased in patients with HF $(0.34 \pm 0.32$; Fig. 1A). Furthermore, the levels of miR-291-3p in fibrosis liver tissues and the normal liver tissues were tested. As shown in Fig. 1B, a much lower miR-291-3p level $(0.42 \pm 0.28)$ was identified in fibrotic liver tissues than that of the normal liver tissues $(1 \pm 0.83)$. In addition, the present study also evaluated whether miR-291-3p present in peripheral blood may be a useful biomarker to determine possible HF in patients. Receiver operating characteristic (ROC) curve analysis showed that peripheral blood miR-291-3p may differentiate patients with HF from healthy controls, with a ROC curve area of 0.942 (95\% confidence interval: 0.858-1.000; $\mathrm{P}<0.001$; Fig. 1C). Meanwhile, the sensitivity and specificity for peripheral blood miR-291-3p were 85.4 and $93.2 \%$, respectively, at the cut-off of 0.208 .

miR-291-3p suppresses TGF- $\beta$ signaling. TGF- $\beta$ signaling pathway has become a novel therapeutic in the prevention and treatment of HF (29). Thus, the effect of miR-291-3p on the TGF- $\beta$ signaling pathway was evaluated. RT-qPCR analysis showed that transfection of miR-291-3p mimic significantly
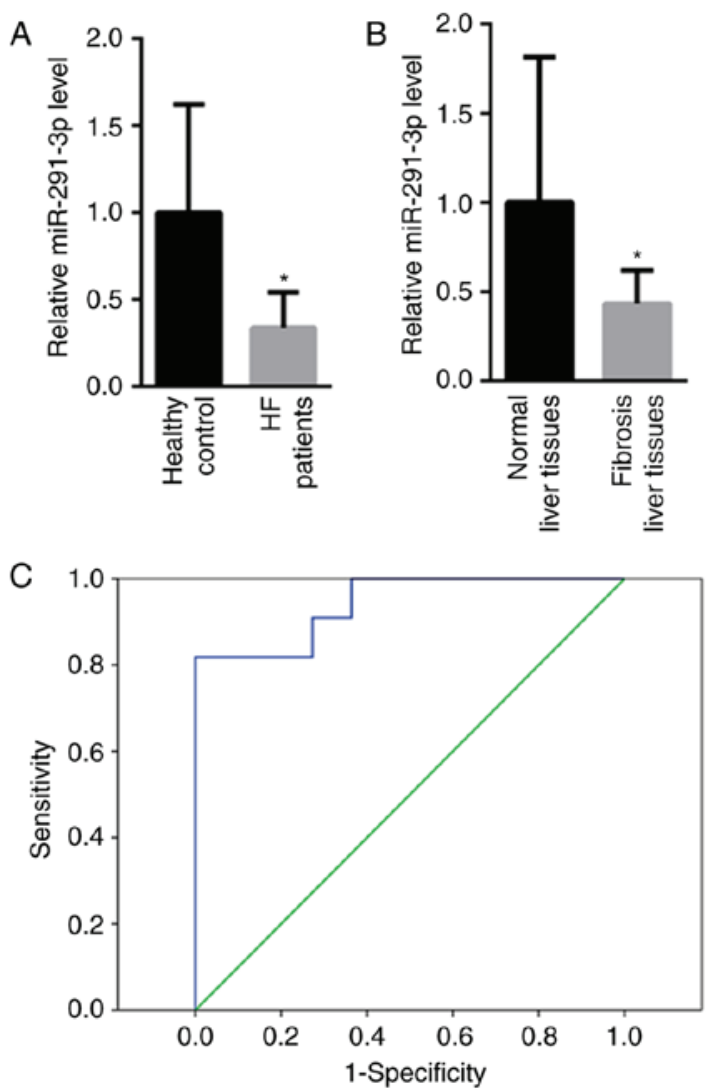

Figure 1. miR-291-3p was decreased in the peripheral blood and liver tissues of patients with HF. (A) Compared with healthy controls, the level of peripheral blood miR-291-3p was decreased in patients with HF. (B) much lower miR-291-3p level was identified in the normal liver tissues compared to that of fibrotic liver tissues. (C) ROC analysis showed that peripheral blood miR-291-3p may differentiate patients with HF from healthy controls. ${ }^{*} \mathrm{P}<0.05$ vs. control. HF, hepatic fibrosis; miR, microRNA; ROC, receiver operating characteristic.

enhanced the level of miR-291-3p $(36.8 \pm 4.5)$ than that of NC (1 \pm 0.12$)$, but suppressed the mRNA levels of Snai1, VE-cadherin, Vimentin, TGF- $\beta 1$, and GFAP (Fig. 2A). In comparison, transfection with miR-291-3p inhibitor decreased miR-291-3p, but increased the mRNA levels of Snai1, VE-cadherin, Vimentin, TGF- $\beta 1$ and GFAP (Fig. 2B). Additionally, the protein expression levels of Snail, VE-cadherin, Vimentin, TGF- $\beta 1$ and GFAP were decreased in HSCs transfected with miR-291-3p mimics (Fig. 2C). Furthermore, the protein expression of Snail, VE-cadherin, Vimentin, TGF- $\beta 1$, and GFAP was also observed to be enhanced in HSCs transfected with miR-291-3p inhibitors compared with that of negative controls (Fig. 2D).

Smad2 is a target gene of miR-291-3p. Based on these above observations, the possible target genes of miR-291-3p were explored. TargetScan analysis demonstrated a conserved binding site of miR-291-3p in the 3'UTR of Smad2 (position 95-119 of Smad2 3'UTR; Fig. 3A). Dual luciferase reporter assay showed that overexpression of miR-291-3p significantly suppressed the relative luciferase activity of pmirGLO-Smad2-3'UTR, but not the relative luciferase activity of pmirGLO-Smad2-3'UTR-Mut (Fig. 3B). Western blot assay also showed that overexpression of miR-291-3p 
A

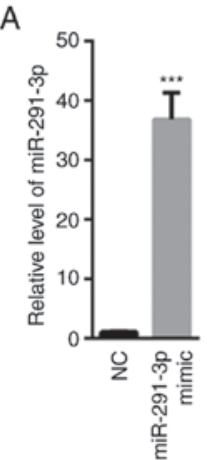

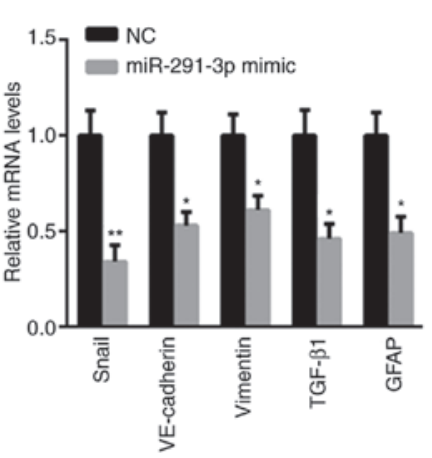
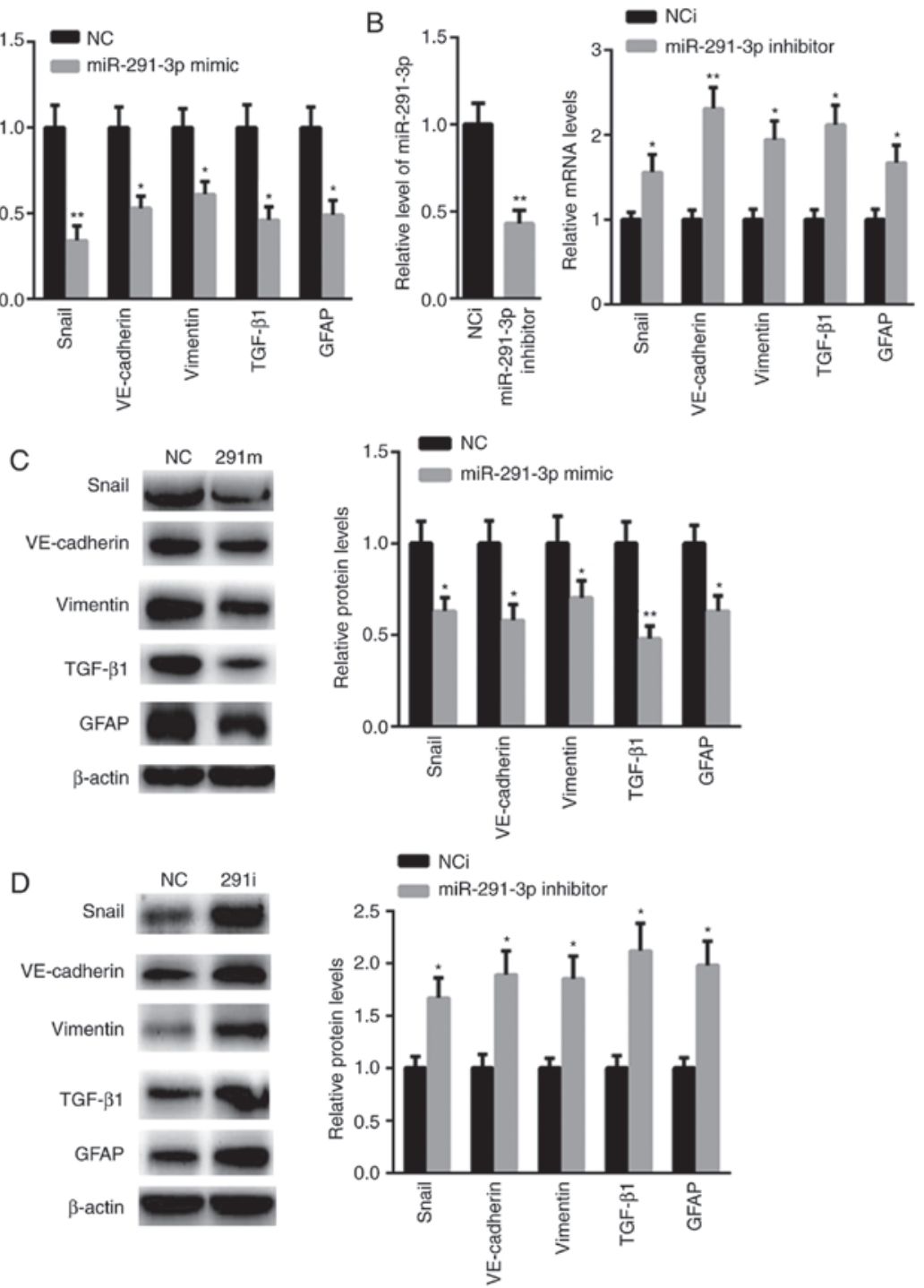

Figure 2. miR-291-3p suppressed TGF- $\beta$ signaling. (A) Reverse transcription-quantitative polymerase chain reaction showed that transfection of the miR-291-3p mimic significantly enhanced the level of miR-291-3p than that of NC (left panel), but significantly suppressed the mRNA levels of Snail, VE-cadherin, Vimentin, TGF- $\beta 1$, and GFAP (right panel). (B) Transfection of miR-291-3p inhibitor decreased miR-291-3p (left panel), but increased the mRNA levels of Snail, VE-cadherin, Vimentin, TGF- $\beta 1$, and GFAP. (C) The protein levels of Snail, VE-cadherin, Vimentin, TGF- $\beta 1$, and GFAP were decreased in HSC transfected with miR-291-3p mimics (right panel). (D) The expression of Snai1, VE-cadherin, Vimentin, TGF- $\beta 1$, and GFAP was also found to be enhanced in HSC transfected with miR-291-3p inhibitors compared to that of negative controls. ${ }^{*} \mathrm{P}<0.05,{ }^{* * *} \mathrm{P}<0.01$ and ${ }^{* * *} \mathrm{P}<0.001$ vs. negative control (NC). GFAP, glial fibrillary acidic protein; HSC, hepatic stellate cells; miR, microRNA; TGF, transforming growth factor; VE-cadherin, vascular endothelial cadherin.

suppressed the expression of Smad2 (Fig. 3C), while its inhibition increased the protein levels of Smad2 (Fig. 3D).

miR-291-3p suppresses TGF- $\beta$ signaling via Smad2. To further evaluate whether miR-291-3p suppressed TGF- $\beta$ signaling via Smad2, a specific siRNA targeting Smad2 was selected. As shown in Fig. 4, silencing of Smad2 significantly inhibited the activation of TGF- $\beta$ signaling. In comparison, inhibition of miR-291-3p increased the activation of TGF- $\beta$ signaling in Fig. 2D. However, knockdown of Smad2 together with miR-291-3p inhibitor, significantly abolished miR-291-3p inhibition-induced activation of TGF- $\beta$ signaling (Fig. 4).

\section{Discussion}

Liver fibrosis is a major characteristic of most chronic liver diseases $(30,31)$. During progression, liver fibrosis can develop into excessive scarring and organ failure, as demonstrated in liver cirrhosis and primary liver cancer (32). It has been suggested that the fibrous ECM is significantly accumulated and increased in the fibrotic liver (33). Hepatic stellate cells are the major contributors for ECM after liver injury. Once HSCs are activated, they become proliferative resulting in enhanced ECM production, thereby leading to liver fibrosis.

Although liver fibrosis can be reversible, how to effectively prevent or reverse the process is still a large challenge for clinicians (34). Additionally, sound diagnostic biomarkers for liver fibrosis are still rare. These limitations largely restrict the treatment options (35). Currently, liver biopsy is the gold standard to identify liver fibrosis (11). However, it is largely questioned due to its painful and invasive characteristics (31). Therefore, early identification of liver fibrosis is of great importance to develop effective antifibrotic therapies. 
A Smad2-3'UTR

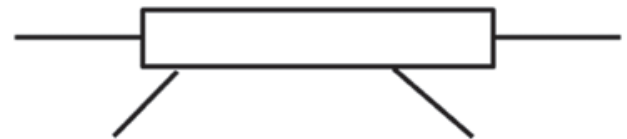

Position 95-119 of Smad2 3'UTR 5'...GUUCAAGAGAGAAAACAGCACUUG

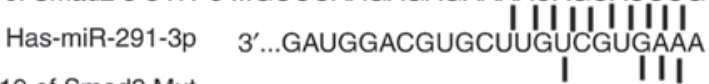
Position 95-119 of Smad2 Mut ${ }_{3}^{\prime}$ '...GUUCAAGAGAGAATTAACTTCUUG
$3^{\prime} U$ TR
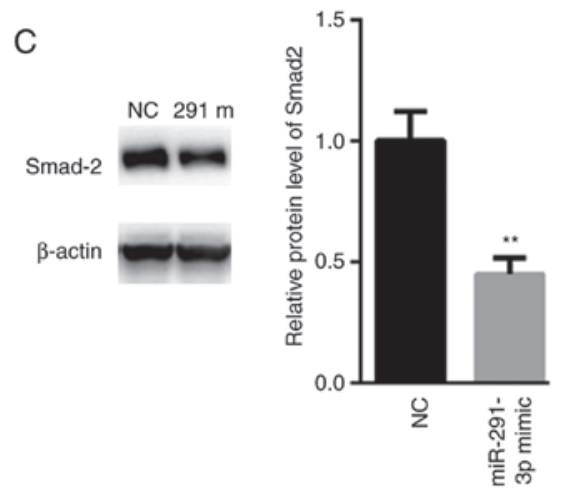
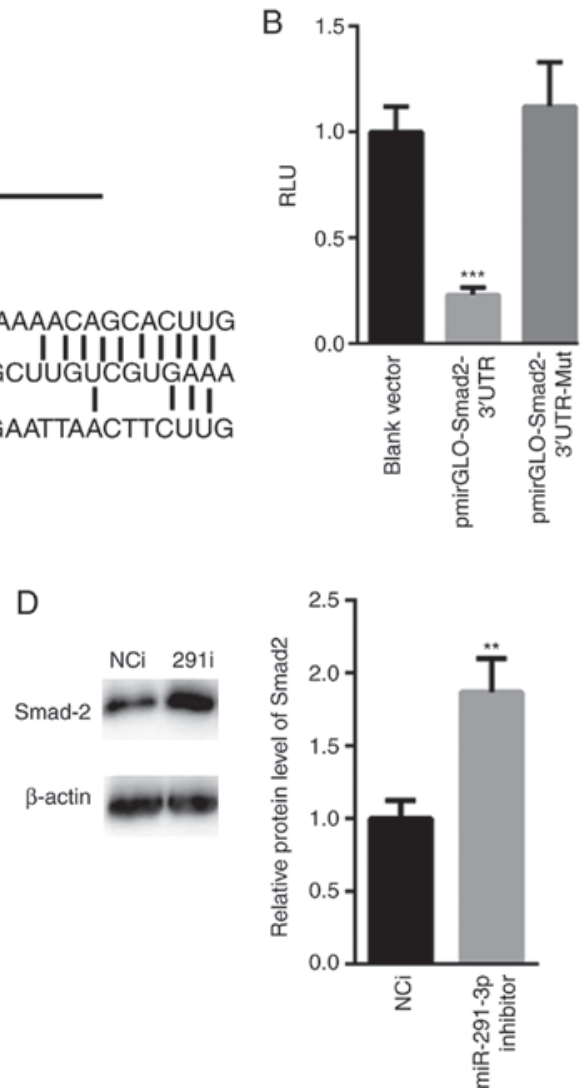

Figure 3. Smad2 is a target gene of microRNA miR-291-3p. (A) TargetScan analysis demonstrated a conserved binding site of miR-291-3p in the 3'UTR of Smad2. (B) Dual luciferase reporter assay showed that overexpression of miRNA miR-291-3p significantly suppressed the relative luciferase activity of pmirGLO-Smad2-3'UTR. Western blot assay showed that the overexpression of miR-291-3p suppressed the (C) Smad2 expression, while the inhibition of miR-291-3p increased the protein level of (D) Smad2. ${ }^{* *} \mathrm{P}<0.01,{ }^{* * *} \mathrm{P}<0.001$ vs. NC. 3'UTR, 3' untranslated region; Smad2, mothers against decapentaplegic homolog 2 ; NC, negative control.
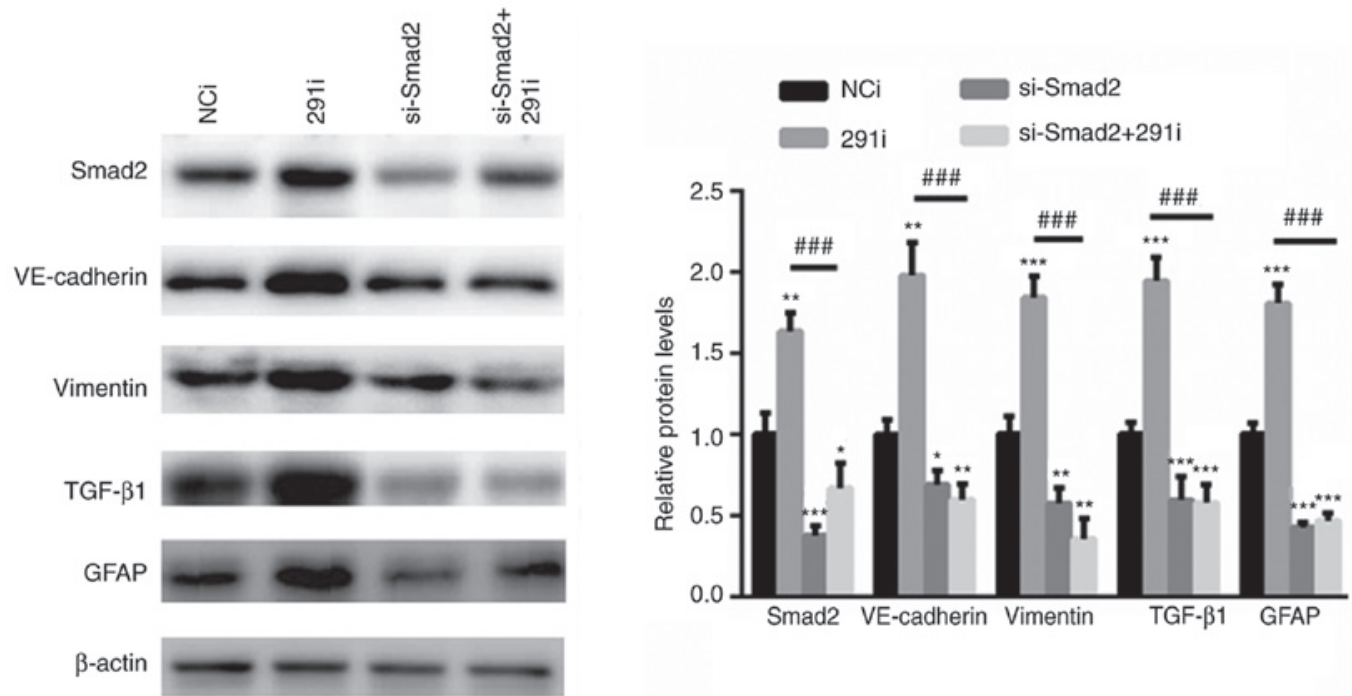

Figure 4. Knockdown of mothers against decapentaplegic homolog 2 Smad2 significantly abolished microRNA miR-291-3p inhibition-induced activation of TGF- $\beta$ signaling in HSCs transfected with miR-291-3p inhibitors compared with that of negative controls. ${ }^{*} \mathrm{P}<0.05,{ }^{* * *} \mathrm{P}<0.01,{ }^{* * *} \mathrm{P}<0.001 \mathrm{vs}$. NC;

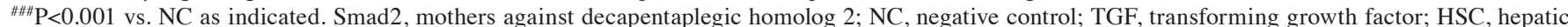
stellate cell; GFAP, glial fibrillary acidic protein; VE, vascular endothelial.

Due to their stability and wide gene-regulating capacity, miRNAs are attractive therapeutic targets $(36,37)$. In the progression of liver fibrosis, increasing evidence has shown the abnormal expression of miRNAs, indicating the importance of maintaining homeostasis via miRNAs $(14,38)$. The present study showed for the first time, to the best of our knowledge, that miR-291-3p was 
decreased in the peripheral blood and liver tissues of patients with HF. ROC analysis showed that miR-291-3p could differentiate patients with $\mathrm{HF}$ from healthy controls.

Based on the above findings, the study further explored the possible association between miR-291-3p and liver fibrosis. TGF- $\beta 1$ is a major cytokine involved in liver fibrosis (39). In the progression of liver fibrosis, TGF- $\beta 1$ could stimulate and activate HSCs to proliferate and constantly secrete ECM via autocrine and paracrine mechanisms, resulting in sustained fibrosis (40). The present study identified that overexpression of miR-291-3p suppressed the activation of TGF- $\beta 1$ signaling, while inhibition of miR-291-3p enhanced TGF- $\beta 1$ signaling activation. Furthermore, it indicated that Smad2 was a target gene of miR-291-3p. More importantly, silencing of Smad 2 could abolish miR-291-3p inhibition-induced TGF- $\beta 1$ signaling activation.

It is true that increased GFAP expression is observed in liver fibrosis compared with normal control (28). However, it has also been stated that high GFAP expression level is associated with low fibrosis (28). These data suggested that GFAP may be a more useful marker than alpha-smooth muscle actin ( $\alpha$-SMA) for early activation of HSCs and represent an early indicator of hepatic fibrogenesis (41). The present study did not examine whether low miR-291 expression is associated with high fibrosis since not enough samples were collected. The expression of GFAP in cultured HSCs and the expression of miR-291-3p was not tested according to the severity of HF was examined. Thus, further study is necessary to explore whether miR-291-3p may represent as an early or late indicator of hepatic fibrogenesis.

At present, the 'gold standard' for diagnosis of liver fibrosis is still liver biopsy, but liver biopsy is an invasive operation, which has some limitations, such as sampling error, subjective deviation of different pathological readers (42). Therefore, non-invasive diagnosis of liver fibrosis has become a research topic in recent years, among which aspartate aminotransferase (AST) to platelet ratio index (APRI) and fibrosis-4 (FIB-4) are widely used (42). However, it is reported that the accuracy of diagnosis of hepatic fibrosis by APRI and FIB-4 alone is not more than $75 \%$ (43). The rate of misdiagnosis and missed diagnosis is still high (43). However, the sensitivity and specificity for peripheral blood miR-291-3p were 85.4 and $93.2 \%$, respectively. The samples in the current study were limited and future work with large patient samples is required to validate the present findings. Studies exploring the diagnostic value of combination of APRI, FIB-4 and peripheral blood microRNA-291-3p for liver fibrosis in patients with chronic hepatitis $B$, are needed in order to improve the diagnostic accuracy of liver fibrosis in patients with chronic hepatitis B.

In summary, reduced peripheral blood miR-291-3p may serve a role in the progression of HF via targeting Smad2. However, there are still some limitations in the current study. On the one hand, the current study was limited due to the small sample size of patient samples. On the other hand, whether miR-291-3p could be the therapeutic target of HF deserves further study.

\section{Acknowledgements}

Not applicable.

\section{Funding}

The present study was supported by a grant from The First People's Hospital of Kunshan Affiliated with Jiangsu University (KAJS-2017052').

\section{Availability of data and materials}

The datasets used and/or analyzed during the current study are available from the corresponding author on reasonable request.

\section{Authors' contributions}

WY performed the experiments, analyzed the data and wrote the mansucript. WZ, YZ, HN and LG performed RT-qPCR experiments. MF designed the experiments, analyzed the data and gave final approval for the publication of the final version of the manuscript. All authors read and approved the final manuscript.

\section{Ethics approval and consent to participate}

The present study was approved by the Ethics Committee of the First Peoples Hospital of Kunshan Affiliated with Jiangsu University and was performed in compliance with the Declaration of Helsinki. All patients have provided written informed consent for this study.

\section{Patient consent for publication}

All patients provided written informed consent for the publication of data in the current study.

\section{Competing interests}

The authors declare that they have no competing interests.

\section{References}

1. Xu B, Zhou NM, Cao WT and Li XJ: Evaluation of elastography combined with serological indexes for hepatic fibrosis in patients with chronic hepatitis B. World J Gastroenterol 24: 4272-4280, 2018.

2. Cargnoni A, Farigu S, Cotti Piccinelli E, Bonassi Signoroni P, Romele P, Vanosi G, Toschi I, Cesari V, Barros Sant'Anna L, Magatti M, et al: Effect of human amniotic epithelial cells on pro-fibrogenic resident hepatic cells in a rat model of liver fibrosis. J Cell Mol Med 22: 1202-1213, 2018

3. Chen J, Li X, Hu Y, Liu W, Zhou Q, Zhang H, Mu Y and Liu P: Gypenosides ameliorate carbon tetrachloride-induced liver fibrosis by inhibiting the differentiation of hepatic progenitor cells into myofibroblasts. Am J Chin Med 45: 1061-1074, 2017.

4. Chen J, Yu Y, Li S, Liu Y, Zhou S, Cao S, Yin J and Li G: MicroRNA-30a ameliorates hepatic fibrosis by inhibiting Beclin1-mediated autophagy. J Cell Mol Med 21: 3679-3692, 2017.

5. Wells RG: The epithelial-to-mesenchymal transition in liver fibrosis: Here today, gone tomorrow? Hepatology 51: 737-740, 2010.

6. Fabris L, Brivio S, Cadamuro M and Strazzabosco M: Revisiting epithelial-to-mesenchymal transition in liver fibrosis: Clues for a better understanding of the 'reactive' biliary epithelial phenotype. Stem Cells Int 2016: 2953727, 2016.

7. Lim YS, Kim KA, Jung JO, Yoon JH, Suh KS, Kim CY and Lee HS: Modulation of cytokeratin expression during in vitro cultivation of human hepatic stellate cells: Evidence of transdifferentiation from epithelial to mesenchymal phenotype. Histochem Cell Biol 118: 127-136, 2002. 
8. Lim YS, Lee HC and Lee HS: Switch of cadherin expression from E- to N-type during the activation of rat hepatic stellate cells. Histochem Cell Biol 127: 149-160, 2007.

9. Ding YF, Wu ZH, Wei YJ, Shu L and Peng YR: Hepatic inflammation-fibrosis-cancer axis in the rat hepatocellular carcinoma induced by diethylnitrosamine. J Cancer Res Clin Oncol 143: 821-834, 2017

10. El-Mezayen NS, El-Hadidy WF, El-Refaie WM, Shalaby TI, Khattab MM and El-Khatib AS: Hepatic stellate cell-targeted imatinib nanomedicine versus conventional imatinib: A novel strategy with potent efficacy in experimental liver fibrosis. J Control Release 266: 226-237, 2017.

11. Shi J, Zhao J, Zhang X, Cheng Y, Hu J, Li Y, Zhao X, Shang Q, Sun Y, Tu B, et al: Activated hepatic stellate cells impair NK cell anti-fibrosis capacity through a TGF- $\beta$-dependent emperipolesis in HBV cirrhotic patients. Sci Rep 7: 44544, 2017.

12. Tang LY,Heller M, Meng Z, Yu LR, Tang Y,Zhou M and Zhang YE: Transforming growth factor- $\beta$ (TGF- $\beta$ ) directly activates the JAK1-STAT3 axis to induce hepatic fibrosis in coordination with the SMAD pathway. J Biol Chem 292: 4302-4312, 2017.

13. Yang JJ, Tao H, Liu LP,Hu W, Deng ZY and Li J: miR-200a controls hepatic stellate cell activation and fibrosis via SIRT1/Notch1 signal pathway. Inflamm Res 66: 341-352, 2017.

14. Zhou G, Lin W, Fang P, Lin X, Zhuge L, Hu Z and Jin L: MiR-10a improves hepatic fibrosis by regulating the TGF $\beta 1 /$ Smads signal transduction pathway. Exp Ther Med 12: 1719-1722, 2016.

15. Wang Y, Du J, Niu X, Fu N, Wang R, Zhang Y, Zhao S, Sun D and Nan Y: MiR-130a-3p attenuates activation and induces apoptosis of hepatic stellate cells in nonalcoholic fibrosing steatohepatitis by directly targeting TGFBR1 and TGFBR2. Cell Death Dis 8: e2792, 2017.

16. Yu F, Chen B, Fan X, Li G, Dong $\mathrm{P}$ and Zheng J: Epigenetically-regulated microRNA-9-5p suppresses the activation of hepatic stellate cells via TGFBR1 and TGFBR2. Cell Physiol Biochem 43: 2242-2252, 2017.

17. Long J, Menggen Q, Wuren Q, Shi Q and Pi X: MiR-219-5p inhibits the growth and metastasis of malignant melanoma by targeting BCL-2. Biomed Res Int 2017: 9032502, 2017.

18. Wang Q, Zhu L, Jiang Y, Xu J, Wang F and He Z: miR-219-5p suppresses the proliferation and invasion of colorectal cancer cells by targeting calcyphosin. Oncol Lett 13: 1319-1324, 2017.

19. Guo J, Li M, Meng X, Sui J, Dou L, Tang W, Huang X, Man Y, Wang S and Li J: MiR-291b-3p induces apoptosis in liver cell line NCTC1469 by reducing the level of RNA-binding protein HuR. Cell Physiol Biochem 33: 810-822, 2014.

20. Meng X, Guo J, Fang W, Dou L, Li M, Huang X, Zhou S, Man Y, Tang W, Yu L and Li J: Liver microRNA-291b-3p promotes hepatic lipogenesis through negative regulation of adenosine 5 '-monophosphate (AMP)-activated protein kinase $\alpha 1$. J Biol Chem 291: 10625-10634, 2016.

21. Guo J, Dou L, Meng X, Chen Z, Yang W, Fang W, Yang C, Huang X, Tang W, Yang J and Li J: Hepatic MiR-291b-3p mediated glucose metabolism by directly targeting p65 to upregulate PTEN expression. Sci Rep 7: 39899, 2017.

22. Weiskirchen R and Gressner AM: Isolation and culture of hepatic stellate cells. Methods Mol Med 117: 99-113, 2005.

23. Livak KJ and Schmittgen TD: Analysis of relative gene expression data using real-time quantitative PCR and the 2(-Delta Delta C(T)) Method. Methods 25: 402-408, 2001

24. Carotti S, Morini S, Corradini SG, Burza MA, Molinaro A, Carpino G, Merli M, De Santis A, Muda AO, Rossi M, et al: Glial fibrillary acidic protein as an early marker of hepatic stellate cell activation in chronic and posttransplant recurrent hepatitis $\mathrm{C}$. Liver Transpl 14: 806-814, 2008

25. Bai X, Saab AS, Huang W, Hoberg IK, Kirchhoff F and Scheller A: Genetic background affects human glial fibrillary acidic protein promoter activity. PLoS One 8: e66873, 2013.

26. Romão LF, Sousa Vde O, Neto VM and Gomes FC: Glutamate activates GFAP gene promoter from cultured astrocytes through TGF-betal pathways. J Neurochem 106: 746-756, 2008

27. Zheng JY, Sun J, Ji CM, Shen L, ChenZJ, Xie P, Sun YZ and Yu RT: Selective deletion of apolipoprotein $\mathrm{E}$ in astrocytes ameliorates the spatial learning and memory deficits in Alzheimer's disease (APP/PS1) mice by inhibiting TGF- $\beta /$ Smad2/STAT3 signaling. Neurobiol Aging 54: 112-132, 2017.
28. Hassan S, Syed S and Kehar SI: Glial fibrillary acidic protein (GFAP) as a mesenchymal marker of early hepatic stellate cells activation in liver fibrosis in chronic hepatitis $\mathrm{C}$ infection. Pak J Med Sci 30: 1027-1032, 2014.

29. Hu Z, Qin F, Gao S, Zhen Y, Huang D and Dong L: Paeoniflorin exerts protective effect on radiation-induced hepatic fibrosis in rats via TGF- $\beta 1 /$ Smads signaling pathway. Am J Transl Res 10: 1012-1021, 2018

30. Jiang Y, Wang C, Li YY, Wang XC, An JD, Wang YJ and Wang XJ: Mistletoe alkaloid fractions alleviates carbon tetrachloride-induced liver fibrosis through inhibition of hepatic stellate cell activation via TGF- $\beta /$ Smad interference. J Ethnopharmacol 158 Pt A: 230-238, 2014.

31. Wang J, Chu ES, Chen HY, Man K, Go MY, Huang XR, Lan HY, Sung JJ and Yu J: microRNA-29b prevents liver fibrosis by attenuating hepatic stellate cell activation and inducing apoptosis through targeting PI3K/AKT pathway. Oncotarget 6: 7325-7338, 2015.

32. Lamb CL, Cholico GN, Pu X, Hagler GD, Cornell KA and Mitchell KA: 2,3,7,8-Tetrachlorodibenzo-p-dioxin (TCDD) increases necroinflammation and hepatic stellate cell activation but does not exacerbate experimental liver fibrosis in mice. Toxicol Appl Pharmacol 311: 42-51, 2016.

33. Liu YW, Chiu YT, Fu SL and Huang YT: Osthole ameliorates hepatic fibrosis and inhibits hepatic stellate cell activation. J Biomed Sci 22: 63, 2015.

34. Nunez Lopez O, Bohanon FJ, Wang X, Ye N, Corsello T, Rojas-Khalil Y, Chen H, Chen H, Zhou J and Radhakrishnan RS: STAT3 inhibition suppresses hepatic stellate cell fibrogenesis: HJC0123, a potential therapeutic agent for liver fibrosis. RSC Adv 6: 100652-100663, 2016.

35. Perumal N, Perumal M, Halagowder D and Sivasithamparam N: Morin attenuates diethylnitrosamine-induced rat liver fibrosis and hepatic stellate cell activation by co-ordinated regulation of Hippo/Yap and TGF- $\beta 1 /$ Smad signaling. Biochimie 140: 10-19, 2017.

36. Zhang Z, Zha Y, Hu W, Huang Z, Gao Z, Zang Y, Chen J, Dong L and Zhang J: The autoregulatory feedback loop of microRNA-21/programmed cell death protein 4/activation protein-1 (MiR-21/PDCD4/AP-1) as a driving force for hepatic fibrosis development. J Biol Chem 288: 37082-37093, 2013.

37. Zhou L, Liu S, Han M, Ma Y, Feng S, Zhao J, Lu H, Yuan X and Cheng J: miR-185 inhibits fibrogenic activation of hepatic stellate cells and prevents liver fibrosis. Mol Ther Nucleic Acids 10: 91-102, 2018.

38. Roderburg C,Luedde M, Vargas Cardenas D, VucurM,Mollnow T, Zimmermann HW, Koch A, Hellerbrand C, Weiskirchen R, Frey N, et al: miR-133a mediates TGF- $\beta$-dependent derepression of collagen synthesis in hepatic stellate cells during liver fibrosis. J Hepatol 58: 736-742, 2013.

39. Xiao YH, Liu DW and Li Q: Effects of drug serum of anti-fibrosis I herbal compound on calcium in hepatic stellate cell and its molecular mechanism. World J Gastroenterol 11: $1515-1520,2005$

40. Liu LX, Zhang HY, Zhang QQ and Guo XH: Effects of insulin-like growth factor binding protein-related protein 1 in mice with hepatic fibrosis induced by thioacetamide. Chin Med J (Engl) 123: 2521-2526, 2010.

41. Zakaria S, Youssef M, Moussa M, Akl M, El-Ahwany E, El-Raziky M, Mostafa O, Helmy AH and El-Hindawi A: Value of $\alpha$-smooth muscle actin and glial fibrillary acidic protein in predicting early hepatic fibrosis in chronic hepatitis $C$ virus infection. Arch Med Sci 6: 356-365, 2010

42. Castera L: Invasive and non-invasive methods for the assessment of fibrosis and disease progression in chronic liver disease. Best Pract Res Clin Gastroenterol 25: 291-303, 2011.

43. Crisan D, Radu C, Lupsor M, Sparchez Z, Grigorescu MD and Grigorescu M: Two or more synchronous combination of noninvasive tests to increase accuracy of liver fibrosis assessement in chronic hepatitis C; results from a cohort of 446 patients. Hepat Mon 12: 177-184, 2012. 